\title{
Spondylocarpotarsal synostosis syndrome due to a novel loss of function FLNB variant: a case report
}

\author{
Samina Yasin ${ }^{1}$, Outi Makitie ${ }^{2,3}$ and Sadaf Naz ${ }^{1 *}$ (D)
}

\begin{abstract}
Background: Loss of function or gain of function variants of Filamin B (FLNB) cause recessive or dominant skeletal disorders respectively. Spondylocarpotarsal synostosis syndrome $(S C T)$ is a rare autosomal recessive disorder characterized by short stature, fused vertebrae and fusion of carpal and tarsal bones. We present a novel FLNB homozygous pathogenic variant and present a carrier of the variant with short height.

Case presentation: We describe a family with five patients affected with skeletal malformations, short stature and vertebral deformities. Exome sequencing revealed a novel homozygous frameshift variant c.2911dupG p.(Ala971GlyfsTer122) in FLNB, segregating with the phenotype in the family. The variant was absent in public databases and 100 ethnically matched control chromosomes. One of the heterozygous carriers of the variant had short stature.

Conclusion: Our report expands the genetic spectrum of FLNB pathogenic variants. It also indicates a need to assess the heights of other carriers of FLNB recessive variants to explore a possible role in idiopathic short stature.
\end{abstract}

Keywords: Carpal synostosis, Scoliosis, Exome sequencing, Pakistan, Case report

\section{Highlights}

- We describe a novel homozygous variant in FLNB

- A biallelic $F L N B$ variant causes SCT in five patients of a family

- Mild short stature of a carrier may indicate a semidominant less severe heterozygous effect of the variant

\section{Background}

Spondylocarpotarsal synostosis syndrome (SCT; OMIM \# 272460) is an autosomal recessive disorder. Patients with SCT are characterized by short stature, vertebral fusion, scoliosis or lordosis and synostosis of carpal and tarsal bones. Other skeletal deformities include

\footnotetext{
* Correspondence: naz.sbs@pu.edu.pk

${ }^{1}$ School of Biological Sciences, University of the Punjab, Quaid-i-Azam Campus, Lahore, Punjab, Pakistan

Full list of author information is available at the end of the article
}

clinodactyly, brachydactyly, limited joint mobility, cleft palate and dysmorphic facial features. Patients with SCT can exhibit dental enamel hypoplasia and mixed hearing loss $[1,2]$.

Filamins arrange actin into three dimensional networks and control structure and function of cytoskeleton. Filamin B, encoded by FLNB is involved in cartilage growth and condensation of developing vertebrae [2]. FLNB is composed of two actin-binding domains $(\mathrm{ABD})$ at the $\mathrm{N}$-terminus and 24 filamin repeat regions. There are two hinges interrupting these repeat regions (Fig. 1) [3].

SCT can be inherited due to $M Y H 3$ variant, or predominantly by biallelic loss of function alleles of $F L N B[1,4]$. Interestingly, heterozygous missense variants of FLNB cause different autosomal dominant syndromes which include Boomerang dysplasia (BD; OMIM \# 112310), Larsen syndrome (LS; OMIM \# 150250), Atelosteogenesis I (AOI; OMIM \# 108720) and III (AOIII; OMIM \# 108721) [2]. 


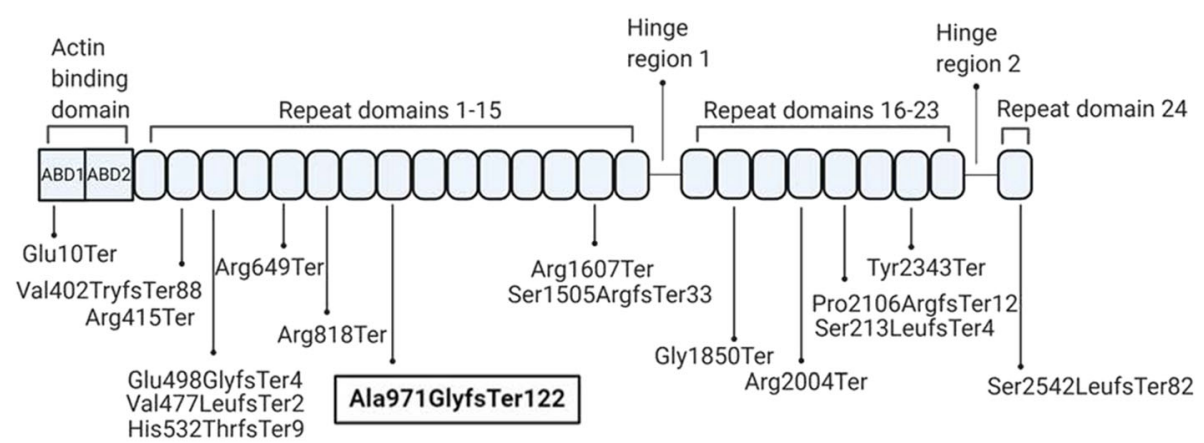

Fig. 1 Schematic representation of the FLNB protein with all identified homozygous variants causing recessive spondylocarpotarsal synostosis syndrome. Variant identified in the present study is shown in bold. ABD1 and ABD2 -Actin binding domains. Twenty-four repeat domains are separated by two hinge regions; hinge 1 and hinge 2

Less than 20 families having patients with FLNB-related SCT are known [3]. We report a large family with SCT due to a novel homozygous frameshift variant of FLNB.

\section{Case presentation}

Subjects and methods

A family SYD07 (Fig. 2a) with two consanguineous couples having five individuals affected with short stature and skeletal malformations was recruited from Punjab. Blood samples were collected from all participants. Genomic DNA was extracted from whole blood according to a standard protocol involving sucrose lysis and salting out.

Exome sequencing was performed on the DNA sample of a severely affected patient III: 1 at $100 \mathrm{X}$ with SureSelect V5-post capture on Illumina platform sequencer (Macrogen, South Korea). WES data was annotated using wANNOVAR (http://wannovar.usc.edu/index. php). Variants were sorted against 1000 g2015, ESP6500 ExAc03 and gnomAD data. Variants with an allele frequency of lower than 0.01 were retained. Homozygous exonic and splice site variants were selected. Candidate variant selected after analysis of exome sequencing data was confirmed by Sanger sequencing of FLNB (NM 001164317.1) exon 20.

Cell lines from the members of family with SCT were not available. Therefore, we obtained blood samples of two affected individuals and one unaffected carrier from family SYD07. RNA was extracted using TRIzol reagent (Thermo Fisher Scientific). Random primed cDNA libraries were synthesized using RevertAid First strand cDNA synthesis kit (Thermo Fisher Scientific). For qualitative PCR, two sets of primers were designed for amplification of partial FLNB cDNA fragments; An Outer 5' - CATC TCAGGAAATCCCCGCCAGCC, $5{ }^{\prime}$-GGTAAGAGAC GGAGCAGGTCCCATC pair and an Inner 5' -CACC CACCCAACAGGGCAACATGC, 5' - GATGGTGAAC TCGGCAGGCTTGCC pair. After PCR with the outer set of primers on cDNA libraries, a nested PCR was performed on the diluted product of $822 \mathrm{bp}$ obtained from the first reaction, using the inner set of primers for a final 458 bp product. GAPDH primer pair of RevertAid First strand cDNA synthesis kit (Thermo Fisher Scientific) was used as a positive control.

For quantitative real time PCR (RT qPCR), one FLNB specific 5' - AATGGGCTGGAAAACAGGGTG, 5' - CAGG TGTCACTAGGCATGGC primer pair and one housekeeping gene GAPDH specific 5' - CGTGGAAGGACTCATG ACCA, 5' - GGATGACCTTGCCCACAGC primer pair were designed. Power SYBR Green Master Mix (Thermo Fisher Scientific) was used for amplification of respective cDNA fragments from an equal input of cDNA libraries of all family members in triplicate on cycler PikoReal 96 (Thermo Fisher Scientific). Each experiment was repeated thrice independently.

\section{Statistical analysis}

Triplicate quantification cycle $(\mathrm{Cq})$ values also called threshold-cycle $(\mathrm{Ct})$ values were averaged and normalised to the control GAPDH values for each sample. Normalised expression of $F L N B$ in the unaffected carrier individual was set to $1 . \Delta \mathrm{Cq}$ and $\triangle \Delta \mathrm{Cq}$ values were calculated to determine the fold change of $F L N B$ in the affected patients. Statistical analysis was done by one-way analysis of variance (ANOVA) Tukey's Multiple Comparison Test to calculate significant fold change ( $p$ values).

\section{Clinical findings}

The five affected individuals ranged in age from 7 to 32 years and their heights were $132 \mathrm{~cm}(5.1 \mathrm{SD}), 125 \mathrm{~cm}$ ($5.0 \mathrm{SD}), 128 \mathrm{~cm}(-4.6 \mathrm{SD}), 119 \mathrm{~cm}(-4.3 \mathrm{SD})$ and $108 \mathrm{~cm}$ (- 3.4 SD) (Table 1). Heights of the parents (II:2, III:8, III: 9) were near or above average $(167 \mathrm{~cm}, 152 \mathrm{~cm}$ and $179 \mathrm{~cm}$ with SDs ranging from -1 to +1 ) except a mother (II:1) who had a height of $147 \mathrm{~cm}(-1.8 \mathrm{SD})$.

Radiographs of patient III:1 revealed fusion of capitate-hamate bones in the hand and tarsal fusion in the feet (Fig. 2b, c). Vertebral radiographs revealed scoliosis, lordosis and disrupted vertebral segmentation (Fig. 

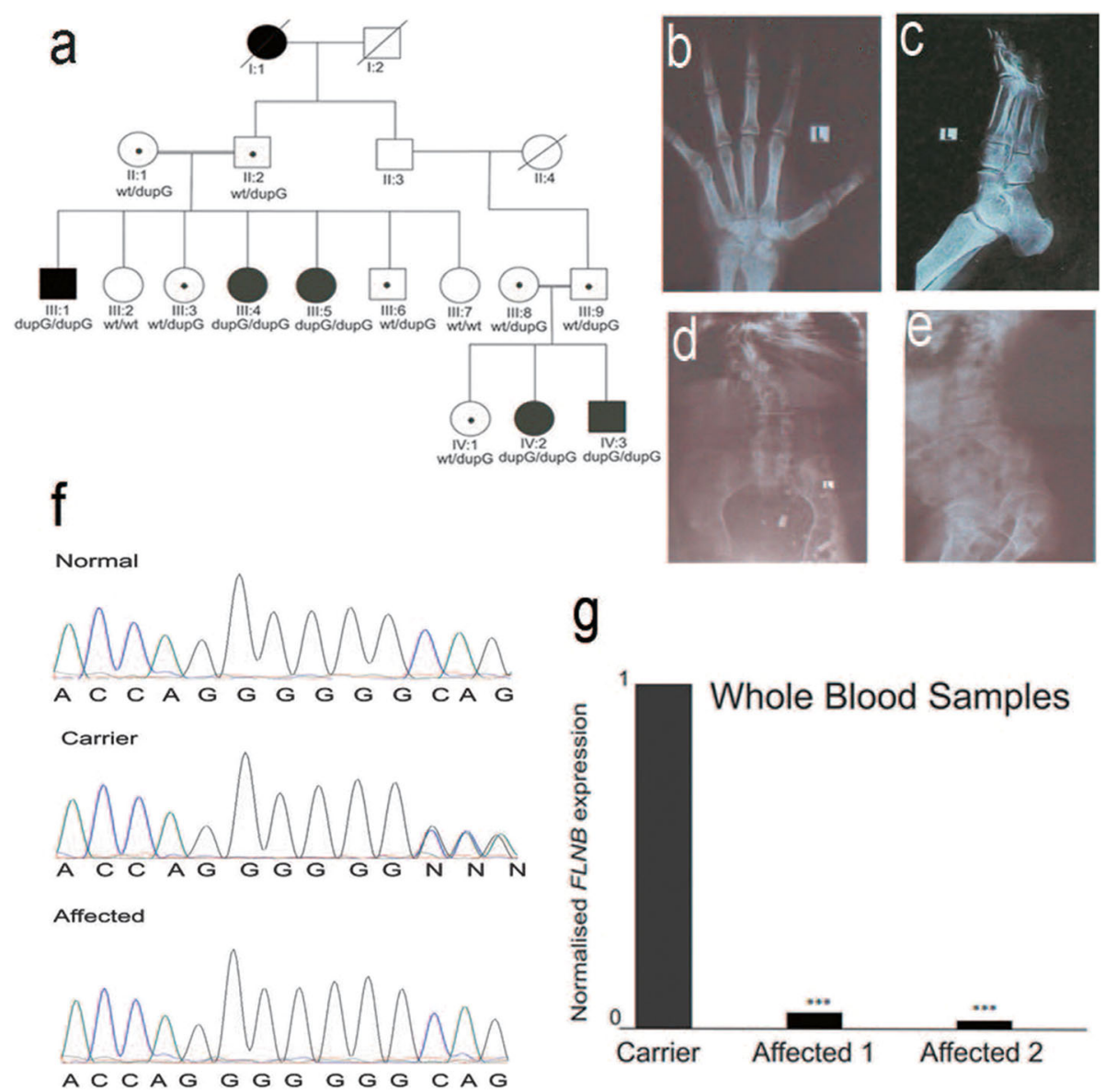

g

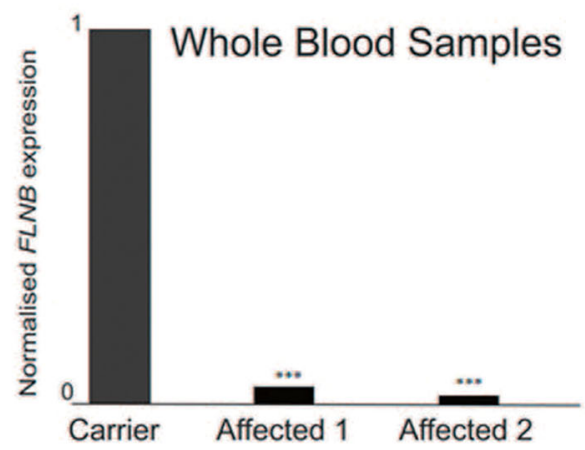

Fig. 2 Pedigree of family SYD07 and radiographs of individual III:1 at 32 years of age. a Pedigree: Black symbols indicate affected individuals. Genotypes of the identified variant for all participants are provided below the symbols. Carriers of the variants are depicted with black dots inside the circles or squares. $\mathbf{b}$ Radiographs of patient III:1: X-Ray of left hand showing capitate hamate (Carpal bones) fusion. $\mathbf{c}$ Anteroposterior view of the left foot showing tarsal fusion. $\mathbf{d}$ Anteroposterior view of the spine showing scoliosis with fusion of vertebrae at thoracic and lumbar region. e Lateral view of the spine showing lordosis. $\mathbf{f}$ Chromatogram of the FLNB showing c.2911dupG variant. Arrows indicate the point of variant from wild type to heterozygous carrier and in homozygous affected individual. $\mathbf{g}$ RT qPCR from whole blood samples of an unaffected heterozygous carrier and two homozygous affected individuals in the family SYD07 demonstrates that the variant c.2911dupG decreases FLNB expression. Significant fold change $(p<0.05, p=0.0003)$ is denoted with three asterisks

2d, e). Neck and trunk were short due to fusion of the vertebrae at cervical and thorax regions (Fig. 2d). The age of onset of visible skeletal malformations was reported to be 7 to 10 years in all affected participants. The early features included short stature and protruding chest. In addition, they had dysmorphic facial features with mild frontal bossing, anteverted nostrils and low set ears, short necks, and spinal deformities (Table 1). Progressive hearing loss, vision impairment, flat feet and limited joint movement were also observed in some of the patients (Table 1).

\section{DNA analysis and RNA expression}

Analysis of exome sequencing data for individual III:1 revealed 34 homozygous variants after applying the filtration criteria (Table S1). We next discarded variants for which a number of individuals were homozygous in the public databases, the affected amino acids were not conserved in different vertebral orthologues or the variants were predicted to be benign by multiple software. This further reduced the number of variants to eight. Among these, the FLNB variant c.2911dupG p.(Ala971GlyfsTer122) (NM 001457.3) segregated with the disease phenotype and was homozygous in all affected members and heterozygous in the obligate carriers (Fig. 2f). This variant was absent from 100 ethnically matched control chromosomes as well as from public databases such as gnomAD (https://gnomad.broadinstitute.org/gene/ ENSG00000136068?dataset=gnomad_r2_1), 1000 genomes (https://www.internationalgenome.org/dataportal/search?q=Ala971GlyfsTer122) GME (http://igm. 
Table 1 Clinical manifestations in affected individual of family SYD07

\begin{tabular}{llllll}
\hline Abnormalities & \multicolumn{3}{l}{ Patients } \\
\cline { 2 - 6 } & III:1 & III:4 & III:5 & IV:2 & IV:3 \\
\hline Age (Years) & 32 & 26 & 24 & 11 & 7 \\
Sex & Male & Female & Female & Female & Male \\
Height (cm) with & 132 & 125 & 128 & 119. & 108 \\
Standard Deviation (SD) & -5.1 & -5.0 & -4.6 & -4.3 & -3.7 \\
Short Stature & + & + & + & + & + \\
Short trunk & + & + & + & + & + \\
Short neck & + & + & + & + & + \\
Hearing deficit & + & + & - & - & - \\
Vision impairment & - & - & + & - & - \\
Dysmorphic face & + & - & - & + & + \\
Flat feet & - & - & - & - & + \\
Joint mobility limitation & + & + & - & - & - \\
\hline
\end{tabular}

(+ present, - absent)

ucsd.edu/gme/data-browser.php) and HGMD (http:// www.hgmd.cf.ac.uk/ac/all.php). The variant has been submitted to the LOVD database (https://databases. lovd.nl/shared/variants/0000600855).

The c.2911dupG variant located within exon 20 creates a frameshift and introduces a premature stop codon, affecting domain 8 of FLNB, and if translated, predicts a 1511 residue shortened protein. Qualitative analysis using nested PCR demonstrated that FLNB specific products could be obtained from cDNA libraries prepared from blood samples of both a heterozygous carrier and the homozygous affected individuals (data not shown). However, quantitative real time PCR revealed that the amount of $F L N B$ specific RNA was significantly reduced $(p<0.05, p=0.0003)$ in both affected individuals as compared to the levels in the unaffected carrier (Fig. 2g).

\section{Discussion and conclusion}

SCT syndrome is a rare disorder involving fusion of vertebrae accompanied by fusion of carpal and tarsal bones. SCT was first recognized in 1973 by Jones et al. with carpal synostosis and fused vertebrae. Other clinical reports revealed autosomal recessive cases comprising of scoliosis along with carpel fusion and block vertebrae [5, 6]. This condition was named as spondylocarpotarsal synostosis syndrome [7] and the causative gene for the syndrome was localized to chromosome 3p14 [8]. Biallelic variants in $F L N B$ underlying this condition were reported in the same year [9]. SCT was described to be caused by truncation of FLNB in its repeat domains or loss of function of the protein. Additional missense, nonsense and frameshift variants were reported subsequently (Table 2) [1, 3, 10, 11].

FLNB is comprised of 24 repeat domains. Autosomal dominant disorders (BD/LS/AOI/AOIII) of FLNB are caused by heterozygous missense variants, which are mostly clustered in actin-binding domains and repeat domains 13-15. They result due to gain-of-function of FLNB. Recently, co-inheritance of some FLNB variants

Table 2 Comparison of clinical features of all patients having biallelic FLNB variants

\begin{tabular}{|c|c|c|c|c|c|c|c|c|c|c|c|}
\hline Study & Variant & $\begin{array}{l}\text { Gender } \\
\text { (Ma:F) }\end{array}$ & $\begin{array}{l}\text { Short } \\
\text { stature }\end{array}$ & $\begin{array}{l}\text { Short } \\
\text { neck }\end{array}$ & $\begin{array}{l}\text { Carpal } \\
\text { fusion }\end{array}$ & Scoliosis & $\begin{array}{l}\text { Facial } \\
\text { deformities }\end{array}$ & $\begin{array}{l}\text { Tarsal } \\
\text { fusion }\end{array}$ & $\begin{array}{l}\text { Hearing } \\
\text { deficit }\end{array}$ & Lordosis & $\begin{array}{l}\text { Enamel } \\
\text { hypoplasia }\end{array}$ \\
\hline $\begin{array}{l}\text { (Krakow et al., } \\
\text { 2004) [9] }\end{array}$ & $\begin{array}{l}\text { c. } 1945 C>T \\
\text { c. } 2452 C>T \\
\text { c. } 4819 C>T \\
\text { c. } 6408 \mathrm{delC} \\
\text { c.7029T }>\mathrm{G}\end{array}$ & $5: 6$ & $11 / 11$ & $9 / 11$ & $11 / 11$ & $11 / 11$ & $11 / 11$ & $7 / 11$ & $7 / 11$ & NR & $7 / 11$ \\
\hline $\begin{array}{l}\text { (Farrington-Rock } \\
\text { et al., 2007) [10] }\end{array}$ & $\begin{array}{l}\text { C. } 4671 G>A \\
\text { c. } 5548 G>T\end{array}$ & $2: 0$ & $2 / 2$ & $2 / 2$ & NR & $2 / 2$ & NR & NR & NR & NR & NR \\
\hline $\begin{array}{l}\text { (Mitter et al., 2008) } \\
\text { [11] }\end{array}$ & c. $6010 \mathrm{C}>\mathrm{T}$ & $1: 0$ & $1 / 1$ & $1 / 1$ & $1 / 1$ & $1 / 1$ & $1 / 1$ & NR & $1 / 1$ & $0 / 1$ & NR \\
\hline $\begin{array}{l}\text { (Yang et al., 2017) } \\
\text { [1] }\end{array}$ & c.7621dupG & $0: 2$ & $2 / 2$ & $2 / 2$ & $2 / 2$ & $2 / 2$ & $0 / 2$ & $0 / 2$ & $1 / 2$ & $2 / 2$ & $0 / 2$ \\
\hline $\begin{array}{l}\text { (Salian et al., 2018) } \\
\text { [3] }\end{array}$ & $\begin{array}{l}\text { c. } 28 \mathrm{G}>\mathrm{T} \\
\text { c. } 429 \text { delinsCT } \\
\text { c.1204delG } \\
\text { c. } 1243 \mathrm{C}>\mathrm{T} \\
\text { c.1493delA } \\
\text { c.1592dup } \\
\text { c.6317delC }\end{array}$ & $6: 4$ & $10 / 10$ & $6 / 10$ & $9 / 10$ & $9 / 10$ & $3 / 10$ & $3 / 10$ & $2 / 10$ & $2 / 10$ & NR \\
\hline Present study & c.2911dupG & $3: 2$ & $5 / 5$ & $5 / 5$ & $4 / 5$ & $3 / 5$ & $3 / 5$ & $3 / 5$ & $1 / 5$ & $3 / 5$ & $0 / 5$ \\
\hline $\begin{array}{l}\text { Summary of } \\
\text { features }\end{array}$ & $\begin{array}{l}\text { All nonsense or } \\
\text { frameshift variants }\end{array}$ & $17: 14$ & $100 \%$ & $93 \%$ & $90 \%$ & $90 \%$ & $54 \%$ & $41 \%$ & $38 \%$ & $22 \%$ & $22 \%$ \\
\hline
\end{tabular}


with variants in other genes important in skeletogenesis, were also identified to have a role in the complex trait of adolescent idiopathic scoliosis [12]. However, the recessively inherited SCT is caused by biallelic missense, nonsense or frameshift truncating variants, which result in loss of function of FLNB [1,13]. Previous studies have shown that SCT is caused generally by variants affecting FLNB actin-binding domains and repeat domains [3] and specifically repeat regions $2,3,5,6,13,14,17,19$, 20, 22, 23 and 24. The novel frameshift variant identified in this study is located within the exon 20 encoding repeat region 8 of FLNB. Frameshift FLNB variants usually result in the absence of the encoded protein, mostly due to nonsense-mediated decay of mRNA [1]. Our results on blood samples also show that the frameshift variant will mark the mRNA for nonsense mediated decay. To our knowledge, this is the first report in which effect of a frameshift variant on FLNB RNA has been determined on a sample directly obtained from SCT patients.

To date 31 genetically characterized patients affected with autosomal recessive SCT from 15 families (including those presented here) harboring 17 biallelic variants of FLNB have been reported (Fig. 1). Clinical symptoms of up to 31 SCT patients have been described in detail $[1,11]$. A short stature, short neck, scoliosis and carpal fusion are exhibited by most of the patients while other disease manifestations are more variable (Table 2). In the current study, the patients exhibited many of the clinical features associated with SCT. They had short statures with short necks and trunks, mild facial dysmorphism and progressive hearing loss. These manifestations were accompanied by scoliosis, lordosis, fusion of vertebrae and synostosis of carpal and tarsal bones. Capitate hamate synostosis was also present.

The less frequent characteristics of SCT include brachydactyly, clinodactyly, club feet, cleft palate and enamel hypoplasia. These conditions have been reported in less than $25 \%$ reported cases $[1,6,14]$. Rarely, rib anomalies, odontoid aplasia and epiphyseal dysplasia with SCT are also present $[1,2,15]$. None of these conditions were manifested by our patients.

In this study, the obligate carriers had the expected heights for their age and sex. However, one female (II:1) who was heterozygous for the variant exhibited mild short stature $(147 \mathrm{~cm},-1.8 \mathrm{SD})$ without other symptoms, indicating a possible association of some FLNB heterozygous variants with milder growth impairment. Previously, heterozygous effect for a loss of function allele has been reported for one obligate carrier who had short stature and unilateral hip dysplasia [11]. It was hypothesized that this could be a nonspecific finding and further research was required. Our work also suggests the need for careful evaluation of carriers of recessively inherited $F L N B$ alleles in order to categorically prove or disprove an incompletely penetrant heterozygous effect of the loss of function variants. Another hypothesis is that some carriers of $F L N B$ variants may have additional variants in other gene/s important for skeletogenesis and an oligogenic mode of inheritance may be responsible for their short stature.

In conclusion, we present a family with autosomal recessive SCT and report a novel frameshift variant which is predicted to result in complete loss of function of FLNB due to nonsense mediated decay of the mRNA. The family has one heterozygous carrier with mild short stature which may indicate a semi-dominant less severe heterozygous effect of the variant or co-inheritance of pathogenic variants in other genes. Our report broadens the genetic spectrum of pathogenic alleles of FLNB.

\section{Supplementary Information}

The online version contains supplementary material available at https://doi. org/10.1186/s12891-020-03890-2.

Additional file 1: Table S1. Variants revealed after whole exome sequence analysis and filtration.

\section{Abbreviations}

SCT: Spondylocarpotarsal synostosis syndrome; ABD: Actin-binding domain; FLNB: Filamin B; SD: Standard deviation; ANOVA: Analysis of variance; RT qPCR: Quantitative Real Time PCR

\section{Acknowledgements}

We thank the participants for their cooperation in blood sampling and radiography. We are grateful to Shereen Gull and Kokab Farooq for generously sharing their knowledge about quantitative PCR.

\section{Authors' contributions}

SN Conceptualization, Supervision, Writing- Original draft preparation. OM: XRays review, Clinical diagnosis and Clinical writing. SY: Investigation, Methodology, Data curation, Writing- Original draft preparation. All authors approved the final manuscript.

\section{Funding}

This research was conducted by funding of Higher Education Commission Pakistan institutional grant to School of Biological Sciences and partial funding by University of the Punjab, Lahore.

The funding organizations were not involved in designing and conducting the study; collection of samples, analysis of the data; preparation of the manuscript; and decision to submit the manuscript for publication.

\section{Availability of data and materials}

The variant number at LOVD data base is 0000600855 (https://databases.lovd. $\mathrm{nl} /$ shared/variants/0000600855). Supplementary data listing filtered variants of exome data is available as Table S1.

Ethics approval and consent to participate

Approval for this study was obtained from the Institutional Review Board of School of Biological Sciences, University of the Punjab, Lahore. Written Informed consents were obtained from all participants and from parents for individuals under 16 years of age.

Consent for publication

Written informed consent to publish was obtained from all participants and from parents for individuals under 16 years of age.

Competing interests

The authors declare that they have no competing interests. 


\section{Author details}

'School of Biological Sciences, University of the Punjab, Quaid-i-Azam Campus, Lahore, Punjab, Pakistan. ${ }^{2}$ Children's Hospital, University of Helsinki and Helsinki University Hospital, Helsinki, Finland. ${ }^{3}$ Folkhälsan Institute of Genetics, Helsinki, Finland.

Received: 11 July 2020 Accepted: 16 December 2020

Published online: 06 January 2021

\section{References}

1. Yang CF, Wang CH, Siong HW, Chang CP, Lin WD, Chen YT, et al. Filamin B loss-of-function mutation in dimerization domain causes autosomalrecessive Spondylocarpotarsal Synostosis syndrome with rib anomalies. Hum Mutat. 2017;38:540-7.

2. Mangaraj S, Choudhury AK, Singh M, Patro D, Baliarsinha AK. Spondylocarpotarsal synostosis syndrome. A rare case of short stature and congenital scoliosis. Clin Cases Miner Bone Metab. 2017:14:258.

3. Salian S, Shukla A, Shah H, Bhat SN, Bhat VR, Nampoothiri S, et al. Seven additional families with spondylocarpotarsal synostosis syndrome with novel biallelic deleterious variants in FLNB. Clin Genet. 2018;94:159-64.

4. Cameron-Christie SR, Wells CF, Simon M, Wessels M, Tang CZ, Wei W, et al. Recessive Spondylocarpotarsal synostosis syndrome due to compound heterozygosity for variants in MYH3. Am J Hum Genet. 2018;102:1115-25.

5. Akbarnia B, Moe J. Familial congenital scoliosis with unilateral unsegemented bar. Case report of two siblings. J Bone Joint Surg. 1978;60:259-61.

6. Coelho KEF, Ramos ES, Felix TM, Martelli L, De Pina-Neto JM, Niikawa N. Three new cases of spondylocarpotarsal synostosis syndrome: clinical and radiographic studies. Am J Med Genet. 1998;77:12-5.

7. Steiner CE, Torriani M, Norato DYJ, Marques-De-Faria AP. Spondylocarpotarsal synostosis with ocular findings. Am J Med Genet. 2000;91:131-4.

8. Steiner C, Ehtesham N, Taylor K, Sebald E, Cantor R, King L, et al. A locus for spondylocarpotarsal synostosis syndrome at chromosome 3p14. J Med Genet. 2004:41:266-9.

9. Krakow D, Robertson SP, King LM, Morgan T, Sebald ET, Bertolotto C, et al. Mutations in the gene encoding filamin B disrupt vertebral segmentation, joint formation and skeletogenesis. Nat Genet. 2004;36:405.

10. Farrington-Rock C, Kirilova V, Dillard-Telm L, Borowsky AD, Chalk S, Rock MJ, et al. Disruption of the Flnb gene in mice phenocopies the human disease spondylocarpotarsal synostosis syndrome. Hum Mol Genet. 2007;17:631-41.

11. Mitter D, Krakow D, Farrington-Rock C, Meinecke P. Expanded clinical spectrum of spondylocarpotarsal synostosis syndrome and possible manifestation in a heterozygous father. Am J Med Genet. 2008;146:779-83.

12. Jiang $\mathrm{H}$, Liang $\mathrm{S}, \mathrm{He} \mathrm{K}, \mathrm{Hu} J, \mathrm{Xu} \mathrm{E}$, Lin $\mathrm{T}$, et al. Exome sequencing analysis identifies frequent oligogenic involvement and FLNB variants in adolescent idiopathic scoliosis. J Med Genet. 2020;57:405-13.

13. Daniel PB, Morgan T, Alanay Y, Bijlsma E, Cho TJ, Cole T, et al. Diseaseassociated mutations in the actin-binding domain of filamin $B$ cause cytoplasmic focal accumulations correlating with disease severity. Hum Mutat. 2012;33:665-73

14. Isidor B, Cormier-Daire V, Le Merrer M, Lefrancois T, Hamel A, Le Caignec C, et al. Autosomal dominant spondylocarpotarsal synostosis syndrome: phenotypic homogeneity and genetic heterogeneity. Am J Med Genet. 2008;146:1593-7.

15. Honeywell C, Langer L, Allanson J. Spondylocarpotarsal synostosis with epiphyseal dysplasia. Am J Med Genet. 2002;109:318-22.

\section{Publisher's Note}

Springer Nature remains neutral with regard to jurisdictional claims in published maps and institutional affiliations.

Ready to submit your research? Choose BMC and benefit from:

- fast, convenient online submission

- thorough peer review by experienced researchers in your field

- rapid publication on acceptance

- support for research data, including large and complex data types

- gold Open Access which fosters wider collaboration and increased citations

- maximum visibility for your research: over $100 \mathrm{M}$ website views per year

At BMC, research is always in progress.

Learn more biomedcentral.com/submissions 